CONCLUSION

\title{
Conclusion: advances in current treatment for patients with newly diagnosed multiple myeloma
}

\author{
S Lonial
}

Leukemia Supplements (2013) 2, S28-S29; doi:10.1038/leusup.2013.6

\section{CONCLUSION}

In recent years, the availability of the novel agents thalidomide, lenalidomide and bortezomib has greatly expanded the treatment options available for the management of patients with newly diagnosed multiple myeloma (NDMM). The articles in this supplement provide an overview of the current approaches to the management of NDMM patients and highlight some of the key research issues that still remain to be answered. The themes regarding the optimal approach to treatment and the role of novel therapies, which emerge from these studies, are summarized below.

An ever increasing number of new induction regimens based on thalidomide, lenalidomide and bortezomib (or combinations of these agents with steroids and alkylating agents) have demonstrated substantial improvements in efficacy and tolerability. ${ }^{1-6}$ The high-quality, durable responses associated with bortezomibor lenalidomide-containing induction regimens are such that some patients may be able to delay the need for autologous stem cell transplantation (ASCT), changing the frontline treatment strategy for NDMM. This approach is being tested in the context of phase III studies with mixed results; thus, it is premature to presume that the high overall response rates seen with new combinations will eliminate the need for ASCT for suitable patients. Currently, all patients who are eligible should be referred to a transplantation center early in their disease course to discuss the timing and collection of stem cell grafts. The role of allogeneic stem cell transplantation remains controversial, with limited use among a majority of patients at this time. Its use is being explored within the setting of clinical trials, but its role in routine care is currently very limited.

Consolidation therapy with novel agents has also been shown to improve outcomes after ASCT, ${ }^{2,7-9}$ and post-ASCT treatment is now a standard approach that should be considered for all patients who do not achieve a complete response to induction and transplantation. However, despite improved induction regimens, tandem ASCT and consolidation treatment, patients with NDMM invariably suffer a relapse. A key aspect of multiple myeloma (MM) treatment strategy is, therefore, the long-term maintenance of disease control. In patients who have had ASCT or in elderly patients ineligible for transplantation, maintenance treatment with thalidomide, ${ }^{10-12}$ lenalidomide ${ }^{13-15}$ or bortezo$\mathrm{mib}^{4,6}$ has demonstrated prolonged clinical remissions and improved control of myeloma. Some evidence suggests improved survival with maintenance strategies, ${ }^{12,14}$ but the optimal approach to maintenance therapy has not been established. The long-term safety profile of lenalidomide makes it an attractive option in this setting, ${ }^{16}$ although additional studies with longer follow-up are needed. The primary adverse events associated with lenalidomide therapy, including myelosuppression, rash, fatigue and venous thromboembolism, are manageable in most cases with standard interventions and dose modifications. Cases of second primary malignancies have been reported in trials of lenalidomide maintenance therapy. ${ }^{13-15}$ Therefore, continued clinical trials and concerted efforts to elucidate the underlying mechanisms and risk factors for this complication are still required.

In conclusion, there have been many recent advances in the treatment of NDMM, and improvement of survival seems to be possible. Clinical trials continue to help define the optimal dose, timing and treatment combinations of each of the novel agents for induction, consolidation and maintenance treatment. As more is learned about the biology of MM, it is increasingly clear that treatment and treatment duration should be individually tailored to each patient's level of risk, based on the presence of adverse genetic prognostic factors (for example, deletion 17p13, deletion $t(4 ; 14))$ as well as the age and clinical condition of the patient. Additional research questions for the future include the timing of ASCT in the era of novel agents, the potential for immunomodulatory drugs to alter the progression of monoclonal gammopathy of undetermined significance or smoldering myeloma to $M M$, and the optimal dose and duration of maintenance treatment with novel agents for long-term disease control, to delay or even prevent relapses in $\mathrm{MM}$ patients who respond to treatment.

\section{CONFLICT OF INTEREST}

SL has had a consultant role for Millennium, Celgene Corporation, Novartis, Merck Bristol Myers Squibb, Onyx and Johnson and Johnson, with honoraria.

\section{ACKNOWLEDGEMENTS}

I thank Shanthi Jayawardena, PhD, and Eva Polk, PhD (Excerpta Medica), for the linguistic improvement of the manuscript. Editorial support in the preparation of this manuscript was funded by Celgene Corporation. SL is supported by the Richard and Annelly Deets Fund for Multiple Myeloma. SL was fully responsible for all content and editorial decisions for this manuscript.

\section{REFERENCES}

1 Rajkumar SV, Jacobus S, Callander NS, Fonseca R, Vesole DH, Williams ME et al Lenalidomide plus high dose dexamethasone versus lenalidomide plus low-dose dexamethasone as initial therapy for newly diagnosed multiple myeloma: an open label randomised controlled trial. Lancet Oncol 2010; 11: 29-37.

2 Cavo M, Tacchetti P, Patriarca F, Petrucci MT, Pantani L, Galli M et al. Bortezomib with thalidomide plus dexamethasone compared with thalidomide plus dexamethasone as induction therapy before, and consolidation therapy after double autologous stem-cell transplantation in newly diagnosed multiple myeloma: a randomised phase 3 study. Lancet 2010; 376: 2075-2085. 
3 Harousseau JL, Attal M, Avet-Loiseau H, Marit G, Caillot D, Mohty M et al. Bortezomib plus dexamethasone is superior to vincristine plus doxorubicin plus dexamethasone as induction treatment prior to autologous stem-cell transplantation in newly diagnosed multiple myeloma: results of the IFM 2005-01 phase III trial. J Clin Oncol 2010; 28: 4621-4629.

4 Sonneveld P, Schmidt-Wolf I, van der Holt B, el Jarari L, Bertsch U, Salwender H et al. Bortezomib induction and maintenance treatment in patients with newly diagnosed multiple myeloma: results of the randomized phase III HOVON-65/ GMMG-HD4 trial. J Clin Oncol 2012; 30: 2946-2955.

5 Richardson PG, Weller E, Lonial S, Jakubowiak AJ, Jagannath S, Raje NS et al. Lenalidomide, bortezomib, and dexamethasone combination therapy in patients with newly diagnosed multiple myeloma. Blood 2010; 116: 679-686.

6 Palumbo A, Bringhen S, Rossi D, Cavalli M, Larocca A, Ria R et al. Bortezomibmelphalan-prednisone-thalidomide followed by maintenance with bortezomibthalidomide compared with bortezomib-melphalan-prednisone for initial treatment of multiple myeloma: a randomized trial. J Clin Oncol 2010; 28: 5101-5109.

7 Mellqvist UH, Gimsing P, Hjertner O, Lenhoff S, Laane E, Remes K et al. Improved progression free survival with bortezomib consolidation after high dose melphalan; results of a randomized phase III trial. Haematologica 2011; 96 (Suppl 1): (abstract O-11).

8 Ladetto M, Pagliano G, Ferrero S, Cavallo F, Drandi D, Santo L et al. Major tumor shrinking and persistent molecular remissions after consolidation with bortezomib, thalidomide, and dexamethasone in patients with autografted myeloma. J Clin Oncol 2010; 28: 2077-2084.

9 Roussel M, Avet-Loiseau H, Moreau P, Huynh A, Benboubker L, Hulin C et al. Frontline therapy with bortezomib, lenalidomide, and dexamethasone (VRD) induction followed by autologous stem cell transplantation, VRD consolidation and lenalidomide maintenance in newly diagnosed multiple myeloma patients: primary results of the IFM 2008 phase II study. Blood 2010; 116: (abstract 624)

10 Lokhorst HM, van der Holt B, Zweegman S, Vellenga E, Croockewit S, van Oers MH et al. A randomized phase 3 study on the effect of thalidomide combined with adriamycin, dexamethasone, and high-dose melphalan, followed by thalidomide maintenance in patients with multiple myeloma. Blood 2010; 115: 1113-1120.

11 Barlogie B, Tricot G, Anaissie E, Shaughnessy J, Rasmussen E, van Rhee F et al. Thalidomide and hematopoietic-cell transplantation for multiple myeloma. $N$ Engl J Med 2006; 354: 1021-1030.

12 Morgan GJ, Gregory WM, Davies FE, Bell SE, Szubert AJ, Brown JM et al. The role of maintenance thalidomide therapy in multiple myeloma: MRC Myeloma IX results and meta-analysis. Blood 2012; 119: 7-15.

13 Attal M, Lauwers-Cances V, Marit G, Caillot D, Moreau P, Facon T et al. Lenalidomide maintenance after stem-cell transplantation for multiple myeloma. N Engl J Med 2012; 366: 1782-1791.

14 McCarthy PL, Owzar K, Anderson KC, Hofmeister CC, Hurd DD, Hassoun $\mathrm{H}$ et al. Lenalidomide after stem-cell transplantation in multiple myeloma. $N$ Engl J Med 2012; 366: 1770-1781.

15 Palumbo A, Hajek R, Delforge M, Kropff M, Petrucci MT, Catalano J et al. Continuous lenalidomide treatment for newly diagnosed multiple myeloma. J Clin Oncol 2012; 366: 1759-1769.

16 Ishak J, Dimopoulos MA, Weber D, Knight RD, Shearer A, Caro JJ. Declining rates of adverse events and dose modifications with lenalidomide in combination with dexamethasone. Blood 2008; 112: (abstract 3708). 